\title{
LA INCLUSIÓN ESCOLAR EN CHILE: OBSERVADA DESDE LA DOCENCIA
}

Nibaldo Benavides-MorenoI

Gril Ortiz-González II

Daniel Reyes-Araya ${ }^{\text {III }}$

I Universidad de Talca, Talca, Maule, Chile; nbenavides@utalca.cl

II Liceo Público Francisco Antonio Encina, Villa Alegre, Maule, Chile; gril.ortiz@gmail.com

III Universidad de Talca, Talca, Maule, Chile; dreyes@utalca.cl

\section{Resumen}

Chile ha impulsado con fuerza terminar con la discriminación en educación en todos sus tipos. Consecuentemente promulgó la Ley de Inclusión Escolar en 2015. Se analizan las opiniones de docentes de establecimientos particulares subvencionados de la región del Maule (Chile), en el ámbito de sus prácticas pedagógicas cotidianas situadas en el marco de esta ley. Es un estudio exploratorio cualitativo. El análisis permite evidenciar el pensamiento y las dinámicas laborales de estos actores en contextos educativos diversos, identificando nudos críticos que de ser atendidos afectarían positivamente su trabajo, observándose la necesidad de corregir dificultades, establecer estrategias pedagógicas y potenciar algunos componentes de la ley.

INCLUSIÓN ESCOLAR • PRÁCTICAS PEDAGÓGICAS • PROFESORES • CHILE

\section{A INCLUSÃO ESCOLAR NO CHILE OBSERVADA A PARTIR DA DOCÊNCIA \\ Resumo}

Com determinação, o Chile tem impulsionado o final de todos os tipos da discriminação educativa. Para tanto, em 2015 promulgou a Lei de Inclusão Escolar. São analisadas as opiniões de professores de estabelecimentos particulares subvencionados da região de Maule (Chile), no âmbito das suas práticas pedagógicas cotidianas no contexto dessa lei. Trata-se de um estudo exploratório qualitativo. A análise permite evidenciar o pensamento e as dinâmicas de trabalho desses atores em contextos educacionais diversos, identificando problemas críticos que, se fossem atendidos, afetariam positivamente seu trabalho, observando-se a necessidade de corrigir dificuldades, estabelecer estratégias pedagógicas e potencializar alguns componentes da lei.

INCLUSÃO ESCOLAR • PRÁTICAS PEDAGÓGICAS • PROFESSORES • CHILE 


\section{SCHOOL INCLUSION IN CHILE: OBSERVED FROM THE TEACHING}

\section{Abstract}

Chile has strongly promoted ending discrimination in education in all its types. Consequently, the Law of School Inclusion was enacted in 2015. The opinions of teachers of subsidized private establishments in the Maule region (Chile) are analyzed, within the scope of their daily pedagogical practices located within the framework of this law. It is a qualitative exploratory study. The analysis makes it possible to demonstrate the thinking and labor dynamics of these actors in diverse educational contexts, identifying critical knots that if taken care of would positively affect their work, observing the need to correct difficulties, establish pedagogical strategies and strengthen some components of the law.

SCHOOL INCLUSION • PEDAGOGICAL PRACTICES • TEACHERS • CHILE

\section{L'INCLUSION SCOLAIRE AU CHILI LE REGARD DES ENSEIGNANTS}

\section{Résumé}

Le Chili est déterminé à mettre fin à toutes les discriminations en matière d'éducation. Pour ce faire, le pays a adopté en 2015 la Lei de Inclusão Escolar [Loi sur l'Inclusion Scolaire]. La recherche analyse les opinions d'enseignants d'établissements privés subventionnés de la région de Maule (Chili), concernant leurs pratiques pédagogiques quotidiennes dans le cadre de cette loi. Il s'agit d'une étude exploratoire qualitative. L'analyse permet de mettre en évidence la pensée et les dynamiques de travail de ces acteurs dans divers contextes éducatifs et d'identifier des problèmes critiques dont la résolution affecterait positivement leur travail. Par ailleurs, elle souligne la nécessité de corriger les difficultés, de mettre en oeuvre des stratégies pédagogiques et d'optimiser certaines composantes de la loi.

INCLUSION SCOLAIRE • PRATIQUES PÉDAGOGIQUES • ENSEIGNANTS • CHILI 
HILE POSEE UNO DE LOS SISTEMAS EDUCATIVOS MÁS SEGREGADOS DEL MUNDO, DONDE LAS oportunidades educacionales de los estudiantes están condicionadas por el nivel socioeconómico, cognitivo, religioso o de comportamiento (Ministério de Educacíon, 2017). En este contexto y con el fin de hacerlo más inclusivo se promulga la Ley de Inclusión Escolar (Ley n. 20.845) en 2015 (entra en vigencia en marzo de 2016), que establece lineamientos para todo establecimiento educacional que reciba dinero del Estado, siendo sus tres ejes fundamentales: fin de la selección de estudiantes, eliminación del financiamiento compartido ${ }^{1}$ (copago de los apoderados) y la prohibición del lucro en educación escolar. Componentes establecidos en la dictadura cívico-militar (1973-1989) y mantenidos e incluso consolidados durante los 90' (Assaél et al., 2011).

En la actualidad el gobierno del presidente Sebastián Piñera declara que esta Ley debe sufrir ajustes, catalogándola de injusta para estudiantes que, por cuestiones ajenas al mérito académico, no pueden ingresar a colegios de su interés, para lo cual propone la Ley de Admisión Justa. ${ }^{2}$ La crítica más álgida, en la voz de la Ministra de Educación de la época, se centra en el Sistema de Admisión Escolar (en adelante $\mathrm{SAE}^{3}$ ), mecanismo que prohíbe la selección por medio de entrevistas, solicitud de calificaciones, pruebas de selección o informes de conducta. Componentes evidentemente discriminatorios y profusamente utilizados por el sistema escolar chileno, que han atentado por décadas contra la igualdad de oportunidades de los estudiantes.

Este escenario no deja lugar a dudas que esta Ley ha sacado a flote todas nuestras sensibilidades, al tenor de que muchas discusiones se han convertido en un campo de batalla ideológico, requiriéndose con urgencia construir caminos efectivos para su mejor desarrollo. Uno de los componentes que evidentemente colabora a despejar interrogantes sobre su contribución -o no- es el docente, puesto que su práctica se ha convertido en un espacio de ejecución y evaluación de dicha política. En el entendido que la diversidad de contextos educativos que debe enfrentar sitúa como foco de atención sus acciones y su formación y, por ende, los alcances de la Ley.

Trabajar en escuelas con población de alta vulnerabilidad, con diversidad cultural, étnica, conductual, lingüística, estudiantes con Necesidades Educativas Especiales (en adelante NEE ${ }^{4}$ ) intelectuales o físicas, genera un espacio de mayor complejidad para el ejercicio docente. Al tenor de esto Ferrada (2015, p. 39) nos señala:

la formación inicial, así como la formación continua de los docentes, aún no han sido abordadas en toda su extensión por las instituciones y agencias encargadas. Esto adquiere especial relevancia cuando estos procesos formativos no distinguen ni han distinguido diversidad de contextos laborales del trabajo docente, es decir, tradicionalmente se ha formado para un contexto laboral homogéneo.

1 Medida que permite a los propietarios privados cobrar un adicional a los apoderados por sobre la subvención que reciben del Estado.

2 Buscaba reinstalar la selección de estudiantes hasta en un 100\% de los cupos "a fin de evaluar el mérito académico" por medio de pruebas o ranking. Los parlamentarios, el 13 de mayo del 2019, por seis votos a favor y siete en contra, rechazaron la idea de debatir en el Congreso esta propuesta; posteriormente, en el mes de julio de 2019, ella se rechazó por parte de la Cámara de Diputados de Chile.

3 Entrega cupos disponibles, salvo algunas prioridades tales como: estudiantes con hermanos en el mismo establecimiento, porcentaje de estudiantes prioritarios, hijos de funcionarios o ex alumnos; a través de un ordenamiento aleatorio de los postulantes, privilegiando el orden de preferencia que las familias identifican en la postulación.

4 Están relacionadas con las ayudas y los recursos especiales que hay que proporcionar a determinados estudiantes que, por diferentes causas - discapacidades, trastornos graves de conducta, incapacidades intelectuales, enfrentan barreras para su proceso de aprendizaje y participación (Organización de las Naciones Unidas para la Educación, la Ciencia y la Cultura [Unesco], 1994) 
Chile implementó en 2017 un nuevo Sistema de Desarrollo Profesional Docente, ${ }^{5}$ lo que plantea la necesidad de una mayor evidencia respecto a los primeros años de docencia en contextos educativos diversos. La realidad docente se vislumbra más compleja que lo que han reflejado los debates. En particular, hay una dimensión cultural que afecta directamente a la tarea docente y está casi ausente en la discusión pública (García-Huidobro, 2016, p. 13). Asimismo, los docentes se ven enfrentados a estudiantes de diferentes características que no acostumbraban acudir a establecimientos particulares subvencionados, estudiantes que por lo general eran atendidos en establecimientos de dependencia municipal (Elacqua, 2009, p. 91). ${ }^{6}$

El artículo analiza las opiniones de docentes de escuelas particulares subvencionadas de la región del Maule (Chile), respecto de algunas dimensiones relevantes en el proceso de implementación de la Ley de Inclusión Escolar, con la finalidad de (i) identificar creencias previas de estos actores en torno a los alcances e impactos que ésta tiene; (ii) precisar demandas pedagógicas, beneficios y dificultades que requieren, por un lado fortalecerse y por otro atenderles y (a partir de todo ello) (iii) formular propuestas de apoyo y de orientación que tiendan a potenciar el trabajo docente en el marco de esta ley.

\section{La educación inclusiva en Chile}

Diferentes convenciones, ${ }^{7}$ declaraciones y tratados en la esfera de los derechos humanos, que Chile ha ratificado, consideran un reconocimiento inalterable hacia la no discriminación, a saber: étnica, género, nivel socioeconómico, religión, conducta, rendimiento. Establecen además que son los sistemas educativos los que deben potenciar la aplicación de procesos inclusivos. Lo que requiere contemplar estrategias y políticas educacionales diferenciadas y complementarias que aseguren la integración de niños y niñas a los sistemas educativos, con capacidad de dar respuesta a la diversidad, expectativas y particularidades (Claro, 2007, p. 3).

La primera atención a la inclusión educativa se encuentra en el ámbito de la integración de estudiantes al sistema regular de educación con alguna NEE. Powell (2015) señala que en Estados Unidos los programas de educación inclusiva acentúan considerablemente los beneficios de la diversidad en el aula para el proceso de aprendizaje intelectual y social de cada individuo con una discapacidad física o cognitiva. Los programas inclusivos de Canadá buscan integrar o ayudar a estudiantes con alguna NEE a cumplir con los objetivos del año académico (Moliner, 2008). Inglaterra quiso abarcar un poco más allá de los programas de integración de niños con NEE, contemplando terminar con la segregación producto de la selección escolar. En este contexto Jenkins, Micklewright y Schnepf (2008) nos señalan que la selección escolar es un factor importante de discriminación, y que los niveles más altos de segregación en las escuelas secundarias estatales se asocian a mayores índices de selección escolar.

En Chile el concepto de inclusión educativa aparece desde el campo de la educación especial y logra imponerse en las políticas públicas en educación; algunos estudios así lo plantean, como Mancebo y Goyeneche (2010). Haciendo un análisis temporal de los niveles de políticas que tenuemente fueron integrando el principio de inclusión debemos remontarnos a la década de los noventa. La Ley Orgánica

5 Creado en abril de 2016 mediante la Leyn. 20.903 (2016); su implementación se lleva adelante entre los años 2017 y 2026. Aborda transformaciones relevantes desde el ingreso a los estudios de pedagogía hasta el desarrollo de una carrera profesional.

6 En Chile, durante la dictadura cívico-militar (1973-1989). se impuso una serie de modificaciones estructurales. En el ámbito educacional las escuelas públicas pasaron a depender de los municipios, se mantuvo el sistema escolar privado creándose además un sistema mixto; los colegios particulares subvencionados, que son establecimientos de propiedad y administración privada, reciben financiamiento estatal vía subvención por estudiante matriculado y efectivamente asistiendo a clase.

7 La relativa a la lucha contra las discriminaciones en la esfera de la enseñanza; el Pacto Internacional de Derechos Económicos, Sociales y Culturales; la Convención sobre la eliminación de todas las formas de discriminación contra la mujer; la Convención de los Derechos del Niño (Organización de las Naciones Unidas [ONU], 1989); y la Agenda de Desarrollo Sostenible de Naciones Unidas (ONU, 2015). 
Constitucional de Enseñanza (1990), que fijó parámetros del sistema educacional por casi 19 años, consideró el concepto de integración, ocupándolo para relacionar al estudiante con su entorno, pero no acorde con su diversidad. En 1994, la Ley n. 19.284 (1994), que estabelece normas para la pelna inegración social de las personas con discapacidad, en materia educacional establece como obligatoria la modalidad de educación diferenciada, incorporando el concepto de adecuación curricular (artículo $27^{\circ}$, p. 8), la mantención de escuelas especiales y la integración de personas con discapacidad en las escuelas ordinarias en todas sus formas (físicas, psíquicas o sensoriales) y plantea como eje articulador el principio de integración. No obstante, solo a partir de 1998 se entregan mayores detalles y se establecen normas acerca de la integración escolar en educación, creando con ello los Proyectos de Integración Escolar (en adelante PIE), ${ }^{8}$ sumándose (en 2005) una Política Nacional de Educación Especial en donde se hace hincapié al trabajo con estudiantes que presenten NEE.

Con lo anterior se promulgan legislaciones como la Ley n. 20248, que establece normas de Subvención Escolar Preferencial (en adelante SEP) ${ }^{9}$ en el 2008 y el Decreto $n .170^{10}$ en 2009 que, si bien demuestran ser un avance al plantear acciones y prácticas destinadas a favorecer el acceso de personas que presenten alguna NEE, no consideran en toda su extensión otras cualidades que conlleva la diversidad. Por tanto, se acuña por primera vez el concepto de inclusión para la educación escolar en la Ley General de Educación, donde se señala

la intención de disminuir la discriminación a una mayor escala es deber del Estado, quien debe velar por la igualdad de oportunidades y la inclusión educativa, promoviendo especialmente que se reduzcan las desigualdades derivadas de circunstancias económicas, sociales, étnicas, de género o territoriales, entre otras. (Ley n. 20.370, 17 de agosto de 2009, pp. 2-3)

Pese a estas iniciativas, en la práctica educativa se crean barreras para la inclusión, en la medida que los establecimientos escolares tienden a la individualización, segregación y a la falta de responsabilidad del aprendizaje de todos sus estudiantes. Estas barreras son culturales, sostenidas por creencias respecto de la educabilidad de los estudiantes, fundamentadas sobre la noción de diversidad como problema y no como recurso (López et al., 2014; M. C. Ferreira \& J. Ferreira, 2004).

La Ley General de Educación se constituye en la antesala para dos de las políticas más importantes en materia de inclusión en Chile: el Decreto n. $83(2015)^{11}$ y la Ley de Inclusión Escolar n. 20.845 en 2015. Esta última impulsa un sistema educativo más inclusivo, que no segregue por condiciones socioeconómicas, culturales o académicas, estableciendo lineamientos concretos para todo establecimiento educacional que reciba dineros del Estado. Los principios claves de la Ley de Inclusión Escolar son:

(i) Integración e inclusión, donde el sistema propenderá a eliminar todas las formas de discriminación arbitraria que impidan el aprendizaje y la participación de los y las estudiantes. Asimismo, el sistema propiciará que los establecimientos educativos sean un lugar de encuentro entre los y las estudiantes de distintas condiciones socioeconómicas, culturales, étnicas, de género, de nacionalidad o de religión; (ii) En los procesos de admisión de los establecimientos subvencionados o que reciban aportes regulares del Estado, en ningún caso se podrá considerar el rendimiento escolar pasado o potencial del postulante. En dichos procesos no será requisito

8 Estrategia inclusiva del sistema escolar, que contribuye al mejoramiento continuo de la calidad de la educación, favoreciendo la presencia en la sala de clases, la participación y el logro de los objetivos de aprendizaje de todos y cada uno de los estudiantes, especialmente de aquellos que presentan NEE, sean éstas de carácter permanente o transitorias (Ministerio de Educación, 2015).

9 Entrega recursos adicionales por cada alumno prioritario (aquellos para quienes las condiciones socioeconómicas de sus hogares pueden dificultar sus posibilidades de enfrentar el proceso educativo) y preferente (aquellos estudiantes que no tengan calidad de alumno prioritario y cuyas familias pertenezcan al $80 \%$ más vulnerable del total nacional.

10 Norma legal que instauró las condiciones para el funcionamiento de los PIE.

11 Fortalece el Decreto n. 170, 14 de mayo de 2009, que estaba relacionado íntegramente a los estudiantes con NEE, a partir de la inclusión curricular de todas las personas en riesgo de ser excluida o discriminada, desde estudiantes con discapacidad física hasta estudiantes extranjeros. 
la presentación de antecedentes socioeconómicos de la familia del postulante, tales como nivel de escolaridad, estado civil y situación patrimonial de los padres, madres o apoderados; (iii) El proceso de admisión de los y las estudiantes que desarrollen los establecimientos que reciben subvención o aportes del Estado se realizará conforme a los principios de transparencia, educación inclusiva, accesibilidad universal, equidad y no discriminación arbitraria, considerando especialmente el derecho preferente de los padres a elegir el establecimiento educacional para sus hijos. (Chile, 2015b, pp. 2-13)

De esta forma Chile en los últimos 27 años ha avanzado de la integración a la inclusión escolar promoviendo leyes, decretos y programas que buscan eliminar cualquier atisbo de discriminación, exclusión o segregación en el ámbito escolar, entregando un rol activo al Estado. Lo que queda como desafío es la consolidación de esta serie de iniciativas, que tengan coherencia con la práctica al interior de las escuelas, y que contribuyan a cambiar y mejorar la cultura escolar, con el objeto de alcanzar una real inclusión educativa, ya que solo así se logrará fortalecer la igualdad de oportunidades en un sistema altamente segregado.

\section{La formación docente para contextos inclusivos}

El proceso de enseñanza/aprendizaje se reconoce esencialmente en el aula, y el factor principal de los resultados de los estudiantes es la dinámica de interacción socioafectiva, cognitiva y pedagógica que ocurre entre el docente y el alumno en el aula y en el centro escolar. Esta relación dual es particularmente relevante cuando nos referimos a un estudiante que presenta condiciones de entrada diversas y/o proviene de familias más carentes y vulnerables (Arellano, 2000; Bellei, 2003; Cox 2001; García-Huidobro \& Cox, 1999). Hacer posible que un profesional ejerza un rol activo en la inclusión educativa es impregnar en los futuros docentes según Fernández (2015, pp. 68-69):

compromiso (voluntad de ayudar a todos los alumnos), afecto (entusiasmo y cariño hacia los alumnos), conocimiento de la didáctica de la materia enseñada (hacerla accesible para todos), múltiples modelos de enseñanza (flexibilidad y habilidad para resolver lo imprevisto), reflexión sobre la práctica y trabajo en equipo que promueva el aprendizaje entre los colegas, e investigación del propio contexto donde se desenvuelve el alumno.

Por tanto, es un desafío para un sistema educativo que sus docentes manejen estrategias pedagógicas y disciplinares adecuadas a ambientes diversos, que aseguren procesos de aprendizaje efectivos, generando un clima aula acorde, de comunicación con afecto y confianza. En un contexto de diversidad complejo como plantea Sotomayor y Dupriez (2007, p. 8):

algunos docentes optan por estrategias de más bajo nivel en el plano de la instrucción, otros fortalecerán su rol mediador, otros docentes verán como primera necesidad entregar a sus estudiantes asistencia social y una acogida efectiva a los niños y niñas, pero sin lugar a dudas poder conjugar estos factores equilibradamente aparece como un elemento central de la competencia docente para la enseñanza.

La falta de adecuación pedagógica refleja la interrogante respecto del tipo de formación que tienen los docentes en otras latitudes. La literatura internacional ha profundizado en esta preocupación (Ee-Gyeong, 2011; Galvis \& Bonilla, 2011; Ingersoll, 1998; Jerald \& Ingersoll, 2002; Lu et al., 2007; Mendonca \& Silva, 2015; Zhou, 2012), llegando incluso a consignar el impacto que ésta tiene en el desarrollo social y en el aprendizaje de los estudiantes. En este escenario Calvo (2013, p. 29), enfatiza el análisis desde Latinoamérica:

la formación de docentes para la inclusión educativa trasciende los programas de formación inicial y amerita programas de formación continua que privilegien el acompañamiento a las prácticas docentes en contextos de vulnerabilidad social. Igualmente requieren procesos de 
sistematización de experiencias en aras de develar "lecciones aprendidas" que puedan traducirse en recomendaciones para la formación de docentes.

La experiencia europea da cuenta de elementos fundantes, asociados al perfil docente a desarrollar para el trabajo de éste en contextos inclusivos. Consecuentemente la Agencia Europea para el Desarrollo de la Educación del Alumnado con Necesidades Educativas Especiales (2011, pp. 10-48) establece algunas reflexiones que resumidamente se presentan:

(i) Valorar la enseñanza inclusiva, donde enfatiza que las diferencias entre estudiantes son un recurso y un valor educativo, y que los docentes deben ser tolerantes a las individualidades y necesidades de sus estudiantes; (ii) Principios de diversidad, donde se señala que la educación se basa en creer en la igualdad, en los derechos humanos y en la democracia para todos los estudiantes, y que la educación inclusiva y la calidad de la educación no pueden tratarse como dos asuntos diferentes; (iii) Promoción del aprendizaje académico, social y emocional del estudiante, donde se enfatiza que todos son igual de importantes, así como el desarrollo de la autonomía y la autodeterminación para todo el alumnado, siendo las expectativas de los profesores una clave determinante para el éxito del estudiante; (iv) Práctica pedagógica, donde finalmente se suscribe que la enseñanza es una actividad que debe resolver problemas, se valora la importancia del desarrollo de una pedagogía personal, siendo la práctica reflexiva un facilitador para conseguir un trabajo eficiente con el equipo de profesores, profesionales y padres de los estudiantes.

Consistente con lo anterior, es importante reconocer primeramente que cuando se piensa, habla y discute sobre el nuevo perfil del educador, existen valores y principios esenciales que deben ser considerados desde su génesis (formación inicial); segundo: una práctica pedagógica coherente con la inclusión educativa, implica dejar de lado la concepción de la pedagogía tradicional (método eminentemente conductista) estableciendo nuevas tareas significativas para detectar necesidades y resolver situaciones que se dan en las aulas inclusivas a través de: (i) una práctica pedagógica más reflexiva, centrada en resolver la multiplicidad de problemas de aprendizaje; (ii) valorar la pedagogía personal (intuición pedagógica) desarrollándola a partir de datos y experiencias que nacen desde la realidad particular; (iii) valorar las dimensiones propuestas por la Agencia como: reconocer que todos los estudiantes están en igualdad de derechos, valorar su diversidad, incentivar el trabajo en equipo, estimular la autonomía de los estudiantes, contar con un desarrollo profesional permanente del profesor, y finalmente como elemento relevante es tener altas expectativas de éxito de todos sus estudiantes.

Sin embargo, una de las barreras principales que impide que un profesor promueva la inclusión en una sala de clases con calidad, es su actitud. Granada et al. (2013, p. 15) la definen como

un conjunto de percepciones, creencias, sentimientos a favor o en contra de la inclusión educativa, y las formas de reaccionar ante la postura educativa que centra su esfuerzo en el logro de los aprendizajes de todos y cada uno de los estudiantes.

Por otro lado, la resistencia al cambio cultural del docente ha sido arraigada a través de la arquitectura institucional y la formación pedagógica inicial centrada en un estudiante homogéneo, lo que acostumbró al profesor a trabajar con estudiantes con cualidades similares, excluyendo al diferente. Realizar este cambio requiere tener el coraje de dejar de usar a la escuela como un instrumento de elitismo y exclusión del conocimiento (Messias et al., 2013).

Finalmente, variados estudios demuestran que un hito a considerar en la formación de docentes para la diversidad, tanto en su formación inicial como continua, es la reconstrucción de una nueva identidad, basada en principios de equidad y justicia, de manera que en el desarrollo profesional docente se configure una cultura profesional con valores más inclusivos, que permita ver a la diversidad como un mecanismo que conlleva a superar desafíos y crea condiciones para la excelencia docente (Blanco, 2009; Correa et al., 2015; Durán \& Climent, 2017; Hsien, 2007; Marchesi, 2001; Moliner \& Loren, 2010; Organización de las Naciones Unidas para la Educación [Unesco], 2009). 
El desafío consiste entonces en colocar en sintonía a las escuelas y, especialmente, a los docentes con las características socioculturales y demandas de formación pretendidas por los estudiantes y sus familias (Fondo de las Naciones Unidas Para La Infancia [Unicef], 2008). Cuestiones inherentes al Sistema de Desarrollo Profesional Docente chileno que se encuentra en marcha, pero vinculado a iniciativas de formación continua demandadas desde lo local. Las políticas de mejora venidas desde esta perspectiva tienden a ser más efectivas.

\section{Metodología}

Se trata de un estudio exploratorio-cualitativo, aplicado a una muestra intencional de 36 docentes de la región del Maule (Chile), que laboran en escuelas particulares subvencionadas, con una experiencia laboral de al menos cuatro años y con al menos dos años de trabajo en la institución educativa participante. Se utilizó una entrevista semi-estructurada validada mediante juicio de expertos. ${ }^{12}$ Por tratarse de un estudio inicial, sus pretensiones son identificar áreas que pudiesen ser investigadas posteriormente; no tiene aspiraciones de representatividad de las temáticas indagadas.

En la fase de levantamiento de información, se contactó telefónicamente a cada uno de los docentes seleccionados, explicando el propósito del estudio e invitándoles a participar. Una vez que se obtuvo el consentimiento, se procedió a calendarizar las entrevistas (julio-agosto 2018) a nivel de establecimiento escolar.

En el trabajo de campo, un investigador entrevistó individualmente a cada actor, conforme al protocolo de entrevista elaborado previamente (Tabla 1). Al inicio, los participantes firmaron un consentimiento informado. El audio de las entrevistas fue grabado, ellas duraron entre 50 y 90 minutos y fueron transcritas para el posterior análisis de contenido. Los resultados fueron ordenados por estamento y unidades de análisis generadas a partir de categorías, transcritas y procesadas con el software NVIVO 10.0.

\section{TABLA 1}

\section{INTERROGANTES Y CATEGORÍAS RESULTANTES}

\begin{tabular}{|c|c|}
\hline Interrogantes utilizadas & Categorías \\
\hline ¿Qué es para usted la Ley de Inclusión Escolar? & $\begin{array}{l}\text { - Acceso } \\
\text { - Oportunidad } \\
\text { - Trato igualitario }\end{array}$ \\
\hline $\begin{array}{c}\text { ¿Cuáles son las principales dificultades que visualiza usted en el } \\
\text { proceso de implementación de la ley? }\end{array}$ & $\begin{array}{l}\text { - Preparación Docente y Recursos } \\
\text { - Desconocimiento de la Ley } \\
\text { - Problemas de aprendizaje } \\
\text { - Desmotivación } \\
\text { - Discriminación } \\
\text { - Apoyo familiar }\end{array}$ \\
\hline $\begin{array}{l}\text { ¿Qué cambios ha realizado en sus prácticas pedagógicas para } \\
\text { atender (integrar) a los estudiantes que ingresan por el SAE? }\end{array}$ & $\begin{array}{l}\text { - Evaluación diferenciada } \\
\text { - Adecuación curricular } \\
\text { - Reforzamiento académico }\end{array}$ \\
\hline $\begin{array}{c}\text { ¿De qué manera la implementación de esta ley ha impactado en } \\
\text { los estudiantes regulares }{ }^{13} \text { del establecimiento? }\end{array}$ & $\begin{array}{l}\text { - Tolerancia } \\
\text { - Conducta escolar } \\
\text { - Desmotivación } \\
\text { - Resultados académicos }\end{array}$ \\
\hline $\begin{array}{l}\text { ¿Qué conocimientos deben adquirir los docentes en su formación } \\
\text { (inicial y continua) para cumplir con esta nueva ley? }\end{array}$ & $\begin{array}{l}\text { - Metodología y técnicas de enseñanzas } \\
\text { - Psicología infanto-juvenil } \\
\text { - Resolución de conflictos }\end{array}$ \\
\hline
\end{tabular}

Fuente: Elaboración propia.

12 Los expertos correspondieron a académicos universitarios especializados en el área de las políticas públicas. Se utilizó el Índice de Validez de Contenido (IVC) de Lawshe (1975).

13 Jóvenes que pertenecen al Establecimiento educacional de origen y que fueron seleccionados para acceder cumpliendo (total o parcialmente) los siguientes requisitos: Entrevistas, solicitud de calificaciones, pruebas de selección y/o informe de conducta. 


\section{Resultados}

Se analizan las opiniones de los docentes en atención a su práctica pedagógica en contextos educativos inclusivos, estableciendo aspectos que deben ser fortalecidos y en otros casos atendidos para potenciar su trabajo en el marco de esta ley.

\section{Creencias sobre el sentido de la ley}

Uno de los principios básicos de la Ley de Inclusión Escolar (2015) es que el sistema educativo propenderá a eliminar todas las formas de discriminación arbitraria que impidan el aprendizaje y la participación de los estudiantes. Coincidente con la categoría con mayor recurrencia (ponderada) por parte de los docentes entrevistados fue Acceso (20 casos, 56\%), comprendida como el término de cualquier tipo de condición (etnia, situación socioeconómica, religión, rendimiento, capacidades cognitivas, comportamiento) que impida el libre acceso a los colegios de un estudiante. Algunas respuestas fueron:

es la aceptación a la diversidad, ya sea en la formación académica, sexualidad y la forma de ser de cada estudiante. La ley incluye a los niños con capacidades diferentes junto a la aceptación de la identidad de género. (entrevistado N. 8).

es una ley que permite a los chiquillos ${ }^{14}$ tener una educación de calidad sin importar su ideologia, su etnia o pensamiento, y trata básicamente de no discriminar por condición socioeconómica, creencia religiosa u orientación sexual. (entrevistado N. ${ }^{\circ}$ ).

En segundo lugar, se sitúa la categoría Oportunidad (12 casos, 35\%) en el entendido de que la Ley ofrece la opción a los individuos a mejores condiciones de equidad y calidad de procesos educativos, en establecimientos educacionales que reciben aportes del Estado. Algunas respuestas señalan:

la Ley busca dar equidad a los procesos educativos chilenos, cambiando la estructura de ingreso a los establecimientos educacionales, de cómo el profesor enseña a estudiantes con capacidades distintas y cómo el alumno aprende en un aula diversa. (entrevistado N. ${ }^{\circ} 10$ ).

busca poder equiparar la admisión, la calidad, la igualdad, regulando esos temas que a nivel social habian quedado un poco desfasados, sobre todo el poder acceder a la educación de manera más transversal e igualitaria. (entrevistado N. ${ }^{\circ}$ ).

Finalmente, aunque en menor recurrencia, los entrevistados aluden a un Trato igualitario (3 casos, 9\%), en el entendido de que primeramente se reconocen habilidades, aptitudes y recursos particulares de cada estudiante y las dificultades (cognitiva o física) propias de cada uno de ellos, remarcando la importancia de atenderlos en un aula común.

ley que asegura educación, sin importar las condiciones que tú traes, en lo cognitivo, socio-afectivo, de género. En el fondo que todos los estudiantes sean tratados por igual. (entrevistado N. ${ }^{\circ} 14$ ). que el colegio sea capaz de trabajar con niños y niñas con ciertas diferencias, dando oportunidad a los estudiantes que tienen una necesidad educativa especial. (entrevistado N. . 26).

En síntesis, frente a las creencias de los docentes de los establecimientos particulares subvencionados, sus opiniones permiten concluir que existen tres componentes con los que mayoritariamente identifican a la Ley de Inclusión; el primero de ellos es la no discriminación, materializada en la finalización a los procesos de selección, permitiendo el acceso abierto sin importar las capacidades individuales, el capital económico, social o cultural del estudiante y su familia; en el segundo, enfatizan una cuestión de justicia al indicar que la ley permite a los estudiantes y sus familias la oportunidad para elegir un establecimiento educativo cercano a sus intereses y necesidades; y en tercero, el fomento a un trato igualitario de estudiantes con capacidades diferentes dentro de las aulas, reconociendo y respetando la diversidad. Sin embargo, en el discurso de los docentes no se visualizan los otros componentes fundantes de la ley, como son la eliminación del copago y el fin al lucro.

14 Es un modismo que se asocia en Chile a niños y jóvenes en edad escolar, siendo una forma coloquial de identificarlos por parte de los adultos. 


\section{Dificultades en el proceso de implementación}

En este contexto se establece una jerarquía de áreas y factores problemáticos. Es así como la categoría de dificultades con más recurrencia fue la Preparación Docente y Recursos (10 casos, 30\%), comprendida como la falta de competencias que poseen los docentes al momento de tratar con estudiantes distintos, que se manifiesta en una falta de medios pedagógicos (que escapan a lo tradicional) que impliquen establecer actividades novedosas que acompañen y faciliten el proceso de enseñanza y aprendizaje de estudiantes diversos, así como un conjunto de servicios e instalaciones adecuadas para desarrollar la actividad educativa. Algunas respuestas representativas fueron:

los profesores estamos poco preparados para recibir alumnos disruptivos o con NEE. No sabemos enfrentar todo tipo de situaciones en el aula. Los materiales son caros y no siempre están. (entrevistado N. 25 ).

no tenemos personal adecuado ni especializado (psicólogos, psicopedagogos asistentes de aula, fonoaudiólogo) que nos ayuden a atender estudiantes con problemas de aprendizaje o disciplinarios. (entrevistado N. 21).

Otro factor problemático aludido por estos actores es el Desconocimiento de la Ley (8 casos, 23\%), debido a la falta de información que emerge del Ministerio de Educación respecto de los alcances, criterios y objetivos que se persigue de la misma, y su coherencia e impacto que tendrá con el Proyecto curricular y el Proyecto Educativo Institucional (en adelante PEI) del establecimiento que se genera en forma autónoma. Reconocen que es una ley integradora, pero señalan no manejar una definición clara acerca de los criterios que se tienen que ocupar en el aula para evaluar e implementar estrategias didácticas, lo que genera desconcierto en algunos docentes:

le falta dar información al Ministerio, yo encuentro que sus integrantes no saben nada. Tenemos un desconocimiento de las repercusiones y consecuencias de la ley. Todos la interpretan a su conveniencia, falta más claridad y masificación de la ley. Falta información y que sea lo más didáctica posible, para que no solo algunos especialistas la entiendan. (entrevistado N. $\left.{ }^{\circ} 10\right)$. muchas veces el enfoque curriculary el reglamento interno que tienen los establecimientos, miradas las exigencias de la ley, no están acordes con la misión y visión de cada colegio, por lo tanto a la hora de tomar alguna decisión chocan esos criterios. (entrevistado N. 9 ).

Otro factor problemático son algunas cualidades (7 casos, 20\%), que presentan algunos estudiantes al ingresar a un nuevo colegio y que se agrupan en dos lineamientos centrales: (a) Problemas de aprendizaje, reflejados en la dificultad de memorizar y comprender contenidos, y el bajo nivel de conocimiento que traen sobre los contenidos previos. Lo que repercute en la capacidad de aprender al ritmo normal de una clase. Algunas respuestas señalan:

lo más problemático en los niños es el deficiente capital cultural y las limitaciones en cuanto a conocimiento obtenido en otros establecimientos. Básicamente estos estudiantes están muy descendidos en comparación con sus compañeros. (entrevistado N. 30 ).

estos niños tienen un aprendizaje más lento que el resto. Demuestran tener problemas cognitivos, son poco inteligentes y no son capaces de resolver una tarea sencilla. (entrevistado N. ${ }^{\circ} 25$ ).

Otra cualidad negativa es (b) la Desmotivación, reflejada en el nulo interés en participar o realizar una actividad pedagógica determinada. Esta indiferencia se traduce en un estado de desconcierto de los docentes afectando su compromiso con la formación del mismo:

generalmente estos niños no quieren aprender, no quieren participar en las actividades. No hay como lograr que participen en las tareas solicitadas, no tienen motivación. (entrevistado N. 9). yo no avanzo tanto con estos niños, porque a muchos no le interesa, disminuyo la cantidad de tareas y no paso todos los contenidos. Vienen con muchas falencias de la casa y de la otra escuela. (entrevistado N. 11 ). 
Un ámbito adicional de dificultades se encuentra en la Discriminación (5 casos, 15\%) que sufren algunos estudiantes que ingresan por el SAE en manos de ciertos miembros de la comunidad escolar ${ }^{15}$ principalmente de familias de estudiantes regulares- al no ser seleccionados, centradas principalmente en características socioeconómicas y/o capital cultural o disciplinario. Lo que evidentemente perjudica el habitual proceso de transición. Algunas respuestas fueron:

no hay aceptación en general por parte de algunas familias, hacen discriminación, me ha pasado muchas veces escuchar: "que ese niño tiene mal comportamiento", "que no corresponde a esta escuela", "que viene de una familia que es más humilde y que no tiene buenos valores y que se mezclará con mi hijo". (entrevistado N. 21).

es el prejuicio de ese estudiante que llega: ¿cómo es?, ¿de dónde viene?, ¿en qué nivel académico está?, ¿cómo es su familia? La no aceptación de la diferencia de culturas de los estudiantes extranjeros por su idioma. (entrevistado N. ${ }^{\circ}$ 34).

Finalmente, una de las últimas dificultades señaladas por los docentes es el Apoyo familiar ( 4 casos, 12\%) que se manifiesta en la falta de preocupación que demuestran con la formación de sus hijos, delegando responsabilidades al establecimiento educacional que no les corresponde. Algunas respuestas:

frente a los problemas de sus hijos tales como: inasistencia, conflictos dentro del colegio; hay familias que les importa muy poco, entonces tenemos más estudiantes abandonados en la escuela. (entrevistado N..$^{\circ}$ ).

se les pide que lleven a sus hijos al departamento de orientación buscando pesquisar sus problemas, para que luego ellos los lleven a profesionales privados y no lo hacen, dejando todo en manos del colegio. (entrevistado N. $\left.{ }^{\circ} 10\right)$.

Lo expuesto evidencia que las dificultades manifestadas por los docentes entrevistados permiten inferir que existen componentes a atender con la llegada de estudiantes diversos a las aulas; primero, su preparación y recursos que se materializa en la falta de habilidades y de condiciones para desarrollar su quehacer profesional eficientemente. Esto demanda potenciar sus competencias pedagógicas tradicionales y de contextos, para poder resolver satisfactoriamente situaciones a las que se enfrentan; segundo, algunas cualidades que traen algunos estudiantes que ingresan por el SAE, producto de su condición de entrada, que se tornan negativas para el desarrollo normal del proceso de enseñanza y aprendizaje, requiriéndose para este efecto estrategias que ayuden a mejorar su motivación, despertando el interés y las ganas de aprender; tercero, el desconocimiento de la Ley -pese a sus dos años de entrada en vigencia- producto de la confusa e inoportuna entrega de información (y asesoría) a cargo de los organismos estatales dispuestos (Superintendencia de Educación ${ }^{16}$ y Ministerio de Educación). Para esto se necesita revisar sus funciones, alcance y metas que explican estos hechos; como cuarta dificultad es la discriminación que sufren los nuevos estudiantes en manos de sus pares (estudiantes regulares), contemplándose la necesidad de revisar las políticas de convivencia de manera que prime el respeto por sobre el enjuiciamiento producto de la diferencia; como última dificultad se encuentra el escaso apoyo familiar en el proceso, lo que demanda fortalecer las capacidades y los vínculos para conseguir una mayor y mejor presencia de éstas.

\section{Cambios en las prácticas pedagógicas}

En este marco, la Evaluación diferenciada (19 casos, 55\%) es la categoría con mayor recurrencia, comprendida como la adecuación que realizan los docentes a los instrumentos de evaluación, producto de las características particulares que traen algunos estudiantes que ingresan por el SAE, y que se centran principalmente en una falta de conocimientos de contenidos previos y/o con NEE. Este

\footnotetext{
15 Conjunto de personas que influyen y son afectadas por un mismo entorno educativo.

16 Organismo creado por la Ley n. 20.529. Su labor es promocionar, informar y educar a todos los integrantes de las comunidades educativas y ciudadanía en general, sobre la normativa educacional y el resguardo de derechos. También, debe dar a conocer los mecanismos disponibles para resolver consultas, solicitar mediaciones y atender denuncias o reclamos (Ley n. 20.529, 27 de agosto de 2011).
} 
acomodamiento en los instrumentos de evaluación consiste en: eliminar preguntas que habitualmente se utilizan, reemplazar el instrumento por uno de menor exigencia y aumentar el tiempo de contestación de los mismos. Algunas respuestas señalan:

realizamos una evaluación acomodada, con menor exigencia y con más tiempo para su contestación, para ser más justos con estos estudiantes. (entrevistado N. ${ }^{\circ}$ ).

mis evaluaciones están enfocadas a atender las diferencias en los estudiantes, para esto tengo escalas distintas. Las pruebas tienen menos cantidad de preguntas y elimino preguntas difíiles. (entrevistado N. 17 ).

Otro cambio es la Adecuación curricular (10 casos, 30\%) que realizan los docentes frente a la problemática y que consiste en realizar cambios y ajustes en los contenidos de las asignaturas, en las planificaciones de clases, en los instrumentos de evaluación, materiales y planes de estudios. Actividades que evidencian que en el fondo los docentes siguen trabajando y enseñando para un estudiante ideal, aquel que no tiene problemas de motivación, aprendizaje ni conductuales, y que frente a la diversidad experimentan ajustes bajando las exigencias académicas.

yo entiendo que estos estudiantes son distintos y por tanto debo hacer una adaptación curricular y evaluativa flexible, dando más tiempo y sacando algunos contenidos menos importantes. (entrevistado N. 8 ).

he ido flexibilizando mis propias formas de organizar los contenidos. Pienso que uno debe adaptar las planificaciones y evaluaciones acorde a los problemas motivacionales que traen. (entrevistado N. 29). Finalmente, la existencia de un Reforzamiento Académico (5 casos, 15\%) es la última adecuación que mencionan los docentes. Es un espacio donde el profesor de la asignatura se reúne con los estudiantes a fortalecer contendidos descendidos, a orientar sobre el desarrollo de hábitos de estudios y de lo trabajado en clases. Estas estrategias planificadas claramente enriquecen la acción educativa y son una medida directa de atención a la diversidad. Algunas respuestas señalan:

se brindan al estudiante horas de refuerzo fuera del horario de clases. El colegio tiene un horario de atención y uno cita a esos niños para ayudarlos y que no tengan que pagar la culpa de venir de malos colegios. (entrevistado N.'2).

implemento permanente deprácticas de ayuda en la sala de clases, prestando más atención al desarrollo de los estudiantes que vienen llegando, para que no queden más atrasados. (entrevistado N. 5 ).

En síntesis, frente a los cambios en las prácticas pedagógicas de los docentes, las opiniones permiten reseñar que existen tres componentes principales; el primero de ellos es la evaluación diferenciada que implementan, y que se materializa con el cambio y adecuación de actividades evaluativas como consecuencia de las características que traen algunos estudiantes que ingresan por el SAE -falta de conocimientos básicos y dificultades para el aprendizaje-; en segundo lugar y bajo la misma premisa los docentes realizan adecuaciones curriculares. Ambas cuestiones conllevan a reflexionar si el camino indicado es acomodar instrumentos de evaluación y reducir o cercenar el currículum frente a la diversidad, o más bien juzgar los acontecimientos y circunstancias en su mérito, ideando otras estrategias que sean más razonables; tercero, implementar refuerzos educativos es un cambio evidentemente positivo y debe trascender en el sistema, de forma que no sean esporádicos o anecdóticos, lo que requiere sistematización.

\section{El impacto de la ley en los estudiantes regulares}

Frente a este componente se establecen dos grupos de categorías, agrupadas en positivo y negativo, siendo la primera la de mayor recurrencia (12 casos, 35\%), y la Tolerancia como su factor más representativo, siendo comprendida como la capacidad que tienen los estudiantes de interactuar y respetar todo tipo de diferencia que puedan tener con sus pares, ya sea en el ámbito cognitivo, sociocultural, conductual, sexual, religioso o físico. Lo que provoca en los estudiantes que ingresan por el SAE un proceso de transición sin tantos sobresaltos, propiciando un mejor clima escolar que deriva en una comunidad escolar menos discriminadora. Algunas respuestas fueron: 
los alumnos antiguos aceptan a los nuevos estudiantes. Veo que ellos implementan muy bien el espiritu de la Ley, al ser capaces de incluirlos y aceptar sus diferencias. Logran ser más abiertos y considerados. (entrevistado N. 12).

[a los estudiantes regulares] los hizo ser más solidarios, más respetuosos y más tolerantes. Veo casos hasta que los protegen cuando están expuestos a hechos negativos. Los nuevos estudiantes tienden a mimetizarse, adoptando las buenas conductas de los antiguos. (entrevistado N. .22).

Desde la perspectiva negativa existe una mayor diversidad de elementos relacionados, siendo la Conducta escolar (10 casos, 28\%) la de mayor recurrencia. Entendida como el deterioro de las relaciones personales, producto del incorrecto comportamiento de algunos estudiantes que ingresan por el SAE al interior de la sala de clases, que impacta negativamente en la cultura de la escuela, generando rechazo en algunos estudiantes regulares.

[estudiantes que ingresan por el SAE] han generado algún tipo de rechazo en los estudiantes antiguos del colegio, dado que provocan mucho desorden en la sala, y ven que los inspectores no saben aplicar el reglamento que maneje el comportamiento de ellos. (entrevistado N. 3).

elproceso de inclusión afecta la cultura de los estudiantes antiguos, generando en primera instancia resistencia al ver el desinterés de los estudiantes nuevos por las tradiciones del colegio. Sienten que se ha "chacreado"17 todo, los niños antes tocaban guitarra en los recreos, hoy venden drogas y marihuana. (entrevistado N. 24).

Otro componente negativo es la Desmotivación (8 casos, 23\%), que demuestran los estudiantes regulares frente a las actividades académicas, dado que algunos estudiantes que ingresan por el SAE, frente a las dificultades académicas, tienen mayores atenciones y favoritismos por parte de los docentes. Expresándose con la adecuación de contenidos y evaluaciones, lo que reditúa en una falta de interés del estudiante regular.

los estudiantes antiguos me reclaman cuando tú haces una evaluación diferenciada, y lo hacen a viva voz diciendo: ¿profesora, por que él tiene un siete y yo también tengo un siete, siendo que yo contesté cuatro preguntas más? (entrevistado N. 2 ).

algunos estudiantes antiguos me preguntan: ¿profesora, de que me sirve tanto esfuerzo si a ellos se les da todo sin hacer nada? Me reclaman que tienen beneficios especiales porque saben menos, y les damos un trato especial debido a que les evaluamos con menos materia. (entrevistado N. ${ }^{\circ} 11$ ).

Finalmente, el último componente negativo, aunque en menor recurrencia, está asociado a la disminución en los Resultados Académicos (5 casos, 14\%), de las pruebas estandarizadas (SIMCE ${ }^{18} \mathrm{y}$ $\mathrm{PSU}^{19}$ ) del colegio respecto del nivel nacional. Cuestión que preocupa a los establecimientos escolares al ver peligrar su prestigio. Algunas respuestas señalan:

va a haber un impacto negativo en los puntajes SIMCE y PSU. Una porque no están preparados y otra porque no les veo interés. Obviamente los rendimientos van a bajar a nivel de escuela y por agregad, o su prestigio. (entrevistado N. 8 ).

con esta ley lamentablemente uno tiene que nivelar hacia abajo, siendo los alumnos antiguos los más perjudicados, debido a que todo el interés está puesto en los más descendidos. (entrevistado N. $\left.{ }^{\circ} 18\right)$.

Se concluye que el impacto en los estudiantes regulares, según los docentes entrevistados, es más positivo que negativo, destacándose la tolerancia como un factor que viene a potenciar en el estudiante regular actitudes que benefician al nuevo integrante, a partir del respeto independientemente de su

\footnotetext{
17 Es un modismo chileno usado para señalar una situación o estado de alteración del orden público o social.

18 Sistema de Medición de la Calidad de la Educación. Corresponde al sistema usado en Chile para la evaluación de aprendizajes. Se creó en 1988 y en la actualidad recopila información sobre rendimiento en pruebas estandarizadas para los grados 2, 4, 6, 8 y 10, además de datos sobre el contexto de enseñanza.

19 Prueba de Selección Universitaria. Es una batería de pruebas estandarizadas, cuyo propósito es la selección de postulantes para la continuación de estudios universitarios en Chile.
} 
condición social, intelectual o física. Acentuar y promover estas prácticas es una tarea relevante del equipo docente y de gestión de la escuela. Sistematizarlas es un beneficio directo a la diversidad; en segundo lugar, y ya en la esfera de lo negativo, el deterioro de la conducta escolar producto de la mala convivencia e indisciplina entre los estudiantes (regular e inclusivo), impacta en la cultura escolar. Requiriéndose del equipo educativo la fijación de programas de convivencia y de atención a factores de riesgos (fracaso académico, problemas familiares, baja autoestima) que desembocan entre otros aspectos nocivos en el uso de sustancias ilícitas; como otro componente negativo le sigue la desmotivación en los estudiantes regulares al visualizar actitudes de privilegios de los docentes frente a los nuevos integrantes, que se manifiestan en el acomodamiento del currículum, generando una falta de entusiasmo en los estudiantes regulares. Es importante revisar estas prácticas en atención a revertir este sentimiento que provoca un desequilibrio evidente en el clima del aula; como último impacto negativo se observa una baja en los rendimientos de las pruebas estandarizadas, lo que implica establecer estrategias didácticas y pedagógicas coherentes con la diversidad (que impliquen su aprovechamiento), armando grupos con diversos integrantes que aporten riqueza y sinergia para favorecer el rendimiento académico y por ende revertir positivamente los resultados en estas evaluaciones externas.

\section{Demandas docentes en el contexto de la ley}

La categoría con más recurrencia fue Metodología y técnicas de enseñanza (19 casos, 55\%) comprendida como la necesidad manifiesta de los docentes en adquirir nuevos recursos pedagógicos que logren cubrir en todos los estudiantes los diferentes estilos y ritmos de aprendizaje, problemas conductuales, diferencias socioculturales y déficit de conocimientos. Permitiendo ofrecer un conjunto de oportunidades lógicamente coordinadas para conseguir determinados objetivos de aprendizajes en sus estudiantes.

tengo que enfrentar distintos tipos de alumnos en mi sala y debo conocer distintas estrategias. Me hace falta un curso de evaluación diferenciada y que te preparen para la diversidad de problemas conductuales y de aprendizaje que se dan en las aulas. Pero no un curso enano que sea un ramo oficial. (entrevistado N. 35 ).

deberiamos tener más prácticas pedagógicas cuando nos formamos como profesores, para conocer todas las realidades de los colegios; yo encuentro que esto es fundamental para conocer los contextos en los cuales mis futuros estudiantes se desarrollan. (entrevistado N. 6 ).

Otra categoría fue Psicología infanto-juvenil (9 casos, 25\%) entendida como la ausencia de conocimientos para enfrentar los principales trastornos sufridos por niños/as y adolescentes, manifestados en la escuela. Los cambios en la vida de un niño pueden afectar su salud mental y por ende su proceso de enseñanza y aprendizaje, haciéndose relevante que los docentes manejen estrategias de atención que les ayuden a entender para poder actuar sobre estos obstáculos.

nosotros vemos convertirse a un niño en adulto, y esos cambios de conducta y de comunicación no sabemos enfrentarlos. Los estudiantes, sobre todo los nuevos, muestran cuadros de ansiedad seria por el cambio de colegio, incluso hay algunos que caen en depresión. (entrevistado N. 32 ). en términos generales, los docentes debemos realizar cursos de psicología infanto-juvenil para poder lidiar con los estudiantes de una manera más cercana, y con las herramientas necesarias para dar apoyo y contención cuando se presentan problemas en la sala de clases. (entrevistado N. ${ }^{\circ}$ ).

Finalmente, la última categoría se asocia a la Resolución de conflictos (7 casos, 20\%), comprendida como la demanda de estrategias para afrontar y resolver distintos problemas entre estudiantes, tanto dentro como fuera del aula. Mantener ambientes sanos favorece el desarrollo normal de una clase y se genera un espacio propicio para la enseñanza y el aprendizaje.

con la llegada de los estudiantes nuevos los ambientes no están tan sanos, y uno debe estar preparado para cosas que pueden pasar. A veces siento que me queda grande porque no sé cómo manejar un conflicto. (entrevistado N. 17). 
debemos tener conocimientos básicos sobre cómo solucionar problemas de indisciplina. Yo sé que existen técnicas para enfrentar a los alumnos conflictivos, pero al menos yo no sé cómo trabajar con ellos. (entrevistado N. 30 ).

En suma, las demandas de habilidades en el contexto de la ley y las opiniones de los docentes permiten concluir que existen tres componentes relevantes. El primero de ellos se relaciona con el mejoramiento de su metodología y técnicas de enseñanza de manera a enfrentar la diversidad en toda su dimensión. Lo que requiere determinar las dificultades de aprendizaje de un estudiante y el origen de sus problemas conductuales, para luego establecer herramientas que permitan atenderlos; en segundo lugar, los docentes demandan una mejor instrucción en psicología infanto-juvenil para enfrentar problemáticas del desarrollo del niño/a que están impactando en su proceso de aprendizaje; como última demanda se encuentra el desarrollo de aptitudes que le permitan resolver conflictos entre estudiantes. En ambos casos se requiere un desarrollo sistemático de capacidades en el docente, lo que hace imprescindible políticas de capacitación pertinentes que les permitan mejorar.

\section{Debates y propuestas}

Los resultados de este trabajo sirven como insumo temprano para que los equipos docentes y de gestión de los establecimientos educativos y organismos estatales (Superintendencia de Educación y Ministerio de Educación) evalúen el proceso de implementación de esta política, en el entendido que contextos educativos inclusivos demandan docentes preparados para trabajar con estudiantes distintos. Lo que requiere diseñar estrategias dirigidas a mejorar su labor y no transformar esta ley en una política errática, que no atiende la realidad y las problemáticas que se presentan en el aula.

Es así como en el plano de las creencias previas de los docentes el énfasis estuvo dado en que la ley de inclusión escolar está orientada a terminar con discriminaciones de todo tipo, eliminando barreras de entrada (intelectual, física, social, económica, cultural) a un establecimiento educacional que recibe fondos públicos. Si un sistema educativo selecciona estudiantes potencia la desigualdad social, por lo tanto es una cuestión de justicia que la ley termine con esta discriminación. No es bueno que un colegio elija a sus estudiantes, más bien son los estudiantes y sus familias los que deben elegir el colegio. Los docentes señalan que esta política promueve la igualdad de trato en las aulas, puesto que su ejercicio debe tener componentes de ecuanimidad frente a las condiciones y obstáculos de los estudiantes. En este sentido se requiere monitorear la operacionalización de la ley en las aulas, pues las dificultades que puede presentar un estudiante que ingresa por el SAE deben tener atención inmediata. Las políticas institucionales tienen mucho que decir y hacer.

Frente a las dificultades, los docentes advierten no sentirse con las habilidades ni con los recursos educativos suficientes para desarrollar su labor. Las condiciones de entrada de algunos estudiantes que ingresan por el SAE -motivación, comportamiento, falta de conocimientos, producto de su situación social, intelectual o física-, la inoportuna asesoría que reciben de los organismos estatales, el rechazo que sufren los estudiantes en manos de sus pares y el escaso apoyo familiar, son componentes que están impactando negativamente en su quehacer pedagógico. Por tanto, no basta con eliminar barreras de entrada para transformar un sistema educativo en inclusivo; se requieren estrategias de atención en las aulas sistematizadas y sensibilizadas por la comunidad escolar, lo que permitirá mejorar los niveles de calidad y equidad.

Relativo a los cambios en las prácticas pedagógicas, los docentes señalan que han aumentado los refuerzos educativos (fortalecer contendidos descendidos y desarrollar hábitos de estudios) con estudiantes que ingresan por el SAE. Como consecuencia del déficit de conocimientos e inconvenientes que algunos de éstos traen, acomodan contenidos y actividades evaluativas que, por cierto, no son las estrategias más pertinentes. Se necesitan acciones que consideren estas condiciones de entrada, ideando tareas y empleando recursos distintos para conseguir logros y metas de aprendizaje. 
En lo referente al impacto que la ley ha tenido en los estudiantes regulares, los docentes destacan la tolerancia y el respeto a las condiciones que presenta un alumno que ingresa por el SAE. En el plano de lo negativo, existe una sensación de que la ley perjudicó la conducta escolar, la motivación del estudiante regular, y los rendimientos en pruebas estandarizadas. Esto requiere establecer un plan de acción con tareas medibles, que reviertan en una progresión temporal dichas percepciones junto a los rendimientos académicos.

En el ámbito de las propuestas de apoyo y de orientación que tiendan a potenciar el trabajo docente en el marco de esta ley es relevante:

(1) Primeramente, fortalecer el Sistema de Desarrollo Profesional Docente, con políticas contextualizadas de formación docente continua, que impliquen mejorar sus competencias para gestionar mejor el currículum (técnicas de enseñanza, estrategias didácticas y evaluativas, psicología infanto-juvenil, técnicas de resolución de conflictos) y establecer una práctica pedagógica reflexiva superior, que valore y considere a la diversidad como un recurso educativo. Para esto, el docente debe estimular e incentivar la autonomía y el trabajo en equipo permanente en sus estudiantes; en segundo lugar, la formación inicial requiere ajustes de mayor significancia, en atención a la falta de preparación que demanda la ley. Se plantean tres ejes fundamentales: (i) metodologías de gestión curricular, que permitan adaptar el trabajo docente conforme a las dinámicas y realidades que se dan en contextos inclusivos; (ii) empoderarse de políticas educacionales que afectan la interacción al interior de las aulas, logrando que el docente sepa diagnosticary proponer acciones pedagógicas a la luz de estas iniciativas; (iii) desarrollar prácticas pedagógicas tempranas basadas en principios de diversidad, requiriendo que el docente aprenda a potenciar la autonomía y la autodeterminación en sus estudiantes, lo que implica que tomen conciencia de que, independientemente de su condición, pueden desarrollar su pleno potencial para el cumplimiento de cualquier meta.

(2) Impulsar pautas de acción para atender, por ejemplo, a una población migrante en aumento (en algunos casos sinser hispanohablantes), estudiantes con NEE (en algunos casos con discapacidad intelectual moderada o grupos numerosos de estudiantes con hiperactividad). Si bien hay avances institucionales al respecto con el Diseño Universal de Aprendizaje (DUA) ${ }^{20}$ y el PIE, lo cierto es que a partir de la puesta en marcha de la ley carecen de efectividad por sus alcances y su alta demanda. Se necesitan iniciativas gubernamentales centradas en un trabajo diferenciado y personalizado, dentro y fuera del aula, individual o grupal. Para esto se debe contar con herramientas de gestión pedagógica como: talleres inclusivos (con participación de representantes de toda la comunidad escolar), adecuaciones curriculares (con evaluación y retroalimentación permanente de los logros académicos conseguidos), un trabajo mancomunado entre los docentes y los equipos multidisciplinarios que integran los PIE (psicopedagoga, educadora diferencial, psicóloga y asistente social), y el apoyo irrestricto de las familias (cartas de compromiso, asistencia a talleres de sensibilización y de apoyo psicosocial para quienes lo requieran).

(3) Promover la existencia de redes entre establecimientos educacionales. Instancias para establecer un trabajo colaborativo entre docentes; para compartir opiniones, tomar decisiones en conjunto, reflexionar sobre sus prácticas pedagógicas y buscar estrategias para dar sustentabilidad a la ley.

\section{Referencias}

Agencia Europea para el Desarrollo de la Educación del Alumnado con Necesidades Educativas Especiales. (2011). Formación del profesorado para la educación inclusiva en Europa: Retos y oportunidades. https://www.european-agency.org/sites/default/files/te4i-synthesis-report-es.pdf

Arellano, J. P. Reforma educacional: Prioridad que se consolida. Editorial Los Andes, 2000. 
Assaél, J., Cornejo, R., González, J., Redondo, J., Sánchez, R., \& Sobarzo, M. (2011). La empresa educativa chilena. Educação e Sociedade, 32(115), 305-322. http://www.scielo.br/pdf/es/v32n115/v32n115a04.pdf

Bellei, C. (2003) ¿Ha tenido impacto la reforma educativa chilena? In C. Cox (ed.), Políticas educacionales en el cambio de siglo (pp. 125-212). Editorial Universitaria.

Blanco, M. R. (2009). La atención educativa a la diversidad: Las escuelas inclusivas. In A. Marchesi, J. C. Tedesco, J. C., \& C. Coll (Coord.), Calidad, equidad y reformas en la enseñanza (p. 87-100). Organización de Estados Iberoamericanos para la Educación, la Ciencia y la Cultura (OEI).

Calvo, G. (2013). La formación de docentes para la inclusión educativa. Páginas de Educación, 6(1), 17-33. http://www.scielo.edu.uy/pdf/pe/v6n1/v6n1a02.pdf

Claro, J. P. (2007). Estado y desafíos de la inclusión educativa en las regiones andina y cono sur. Revista Iberoamericana sobre Calidad, Eficacia y Cambio en Educación, 5(5), 179-187. http://www.redalyc.org/ articulo.oa?id=55121025026

Correa, J., Sierra, M., \& Alzate, G. (2015). Formación de docentes participantes en el programa de educación inclusiva con calidad en Colombia. Revista Latinoamericana de Educación Inclusiva, 9(1), 43-61. http://www.rinace.net/rlei/numeros/vol9-num1/art2.pdf

Cox, C. (2001). El esfuerzo de reforma del sistema educativo escolar: Siete puntos para una reflexión docente. Santiago de Chile: Mineduc.Decreto n. 170, 14 de mayo de 2009. Fija normas para determinar los alumnos con necesidades educativas especiales que serán beneficiarios de las subvenciones para educación especial. Santiago de Chile.

Decreto n. 83, 30 de enero 2015. Aprueba criterios y orientaciones de adecuación curricular para estudiantes con necesidades educativas especiales de educación parvularia y educación básica. Santiago de Chile.

Durán, D., \& Climent, G. (2017). La formación del profesorado para la educación inclusiva: Un proceso de desarrollo profesional y de mejora de los centros para atender la diversidad. Revista Latinoamericana de Educación Inclusiva, 5(2), 153-170. http://repositoriocdpd.net:8080/handle/123456789/1913

Ee-gyeong, k. (2011). Out-of-field secondary school teachers in Korea: Their realities and implications. KEDI Journal of Educational Policy, 8(1), 29-48. https://search.proquest.com/ docview/1013971203? accountid $=14675$

Elacqua, G. (2009). The impact of school choice and publicpolicy on segregation: Evidence from Chile. Santiago de Chile: Centro de Políticas Comparadas en Educación, Universidad Diego Portales.

Fernández, J. M. Desafíos de la formación docente ante la exclusión educativa. In L. Ortiz, A. Sánchez, A. Luque, \& V. Figueredo. (Coords.), Espacios para hablar y compartir sobre la intervención social y educativa en grupos vulnerables (pp. 63-70). Enfoques Educativos SL, 2015.

Ferrada, D. (2015). El ejercicio docente en contextos de alta vulnerabilidad y diversidad territorial, cultural y lingüística. Revista Docencia, 56, 36-46. http://revistadocencia.cl/ revist37/web/images/ediciones/ Docencia_56.pdf

Ferreira, M. C., \& Ferreira, J. (2004). Sobre inclusão, políticas públicas e práticas pedagógicas. In M. Góes, \& A. Laplane. (Orgs.), Políticas e práticas de educação inclusiva (pp. 21-48). Autores Associados.

Fondo de las Naciones Unidas para la Infancia. (2008). Escuela e Inclusión, círculo virtuoso para la experiencia escolar. Aprendizajes del Seminario Latinoamericano "Prevención de la deserción escolar, reinserción e inclusión educativa". LOM Ediciones. https://unicef.cl/web/escuela-e-inclusion-circulo-virtuosopara-la-experiencia-escolar-aprendizajes-del-seminario-latinoamericano-prevencion-de-la-desercionescolar-reinsercion-e-inclusion-educativa-2/

Galvis, L., \& Bonilla, L. (2011). Desigualdades regionales en el nivel educativo de los profesores en Colombia. Revista de Economia Institucional, 14(26), 223-240. https://www.economiainstitucional.com/pdf/ No26/lgalvis.pdf

García-Huidobro, J. E., \& Cox, C. (1999). La reforma educacional chilena 1990-1998 - la reforma educacional chilena: Visión de conjunto. In García-Huidobro, J. E. (Ed.), La reforma educacional chilena (pp. 7-46). Editorial Popular. 
García-Huidobro, J. C. (2016). Primeros años de docencia en contextos de pobreza: Preguntas que la evidencia desde EE.UU sugiere para la conversación en Chile. Pensamiento Educativo: Revista de Investigación Educacional Latinoamericana, 53(1), 1-16. http://pensamientoeducativo.uc.cl/index.php/pel/article/ view/783/1510

Granada, M., Pomés, M., \& Sanhueza, S. (2013). Actitud de los profesores hacia la inclusión educativa. Papeles Trabajo, 25, 51-59. http://hdl.handle.net/2133/3301

Hsien, M. (2007). Teacher attitudes towards preparation for inclusion: In support of a unified teacher preparation program. Postgraduate Journal of Education Research, 8(1), 49-60.

Ingersoll, R. (1998). The problem of out-of-field teaching. Phi Delta Kappan, 79(10), 773-776. https://repository. upenn.edu/gse_pubs/137/

Jenkins, S., Micklewright, J., \& Schnepf, S. (2008). Social segregation in secondary schools: How does England compare with other countries? Oxford Review of Education, 34(1), 21-37. https://www.tandfonline. com/doi/abs/10.1080/03054980701542039. 2019

Jerald, C., \& Ingersoll, R. (2002, agosto). All talk, no action: Putting an end to out-of-field teaching. The Education Trust, 1-14. https://repository.upenn.edu/gse_pubs/142/

Lawshe, C. H. (1975). A quantitative approach to content validity. Personnel Psychology, 28, 563-575.

Ley n. 19.284, 5 de enero de 1994. Establece normas para la plena integracion social de personas con discapacidad. Santiago de Chile.

Ley n. 20.248, 25 de enero de 2008. Establece Ley de Subvención Escolar Preferencial. Santiago de Chile.

Ley n. 20.370, 17 de agosto de 2009. Establece la Ley General de Educación. Santiago de Chile.

Ley n. 20.529, 27 de agosto de 2011. Sistema Nacional de Aseguramiento de la Calidad de la Educación Parvularia, Básica y Media y su Fiscalización. Santiago de Chile.

Ley n. 20.845, 29 de mayo de 2015. De inclusión escolar que regula la admisión de los y las estudiantes, elimina el financiamiento compartido y prohíbe el lucro en establecimientos educacionales que reciben aportes del Estado. Santiago de Chile, 2015b.

Ley n. 20.903, 01 de abril de 2016. Crea el Sistema de Desarrollo Profesional Docente y modifica otras normas. Santiago de Chile, 2016.

Ley Orgánica Constitucional, 7 de marzo de 1990. Ley Orgánica Constitucional de Enseñanza. Santiago de Chile. López, V., Julio, C., Pérez, M., Morales, M., \& Rojas, C. (2014). Barreras culturales para la inclusión: Políticas y prácticas de integración en Chile. Revista de Educación, 363, 1-17. http://www.revistaeducacion. educacion.es/doi/363_180.pdf

Lu, X., Shen, J., \& Poppink, S. (2007). Are teachers highly qualified? A National Study of Secondary Public School Teachers Using SASS 1999-2000. Leadership and Policy in Schools, 6(2), 129-152. https://doi. org/10.1080/15700760601168636

Mancebo, M., \& Goyeneche, G. (2010, diciembre 6, 9 y 10). Laspolíticas de inclusión educativa: Entrela exclusión social y la innovación pedagógica. In Jornadas de Sociología de la UNLP, Facultad de Humanidades y Ciencias de la Educación, Universidad Nacional de La Plata, Buenos Aires, Argentina. http://www. memoria.fahce.unlp.edu.ar/trab_eventos/ev.5273/ev.5273.pdf

Marchesi, A. (2001). La práctica de las escuelas inclusivas. In J. Palacios, A. Marchesi, \& C. Coll (Comp.), Desarrollo psicológico y educación: Trastornos del desarrollo y necesidades educativas especiales (Vol. 3, pp. 45-70). Alianza.

Mendonca, F., \& Silva, D. (2015, setembro). A formação docente no contexto da inclusão: Para uma nova metodologia. Cadernos de Pesquisa, 45(157), 508-526. http://www.scielo.br/scielo.php?script=sci_ arttext\&pid=S0100-15742015000300508\&lng $=e s \& n r m=i s o$

Messias, V. L., Muñoz, Y., \& Lucas-Torres, S. (2013). Apoyando la inclusión educativa: Un estudio de caso sobre aprendizaje y convivencia en la educación infantil en Castilla-La Mancha. Revista Latinoamericana de Educación Inclusiva, 6(2), 25-42. https://dialnet.unirioja.es/servlet/articulo?codigo= 4268402 
Ministerio de Educación. (2015). Antecedentes generales PIE. Santiago de Chile. https://www.ayudamineduc. $\mathrm{cl} /$ ficha/antecedentes-generales-pie-5\#: :text=El\%20PIE\%20es\%20una\%20estrategia,de\%20 aprendizaje $\% 20 \mathrm{de} \% 20$ todos\%20y

Ministerio de Educación. (2017). El primer gran debate de la reforma educacional: Ley de inclusión escolar.

Moliner, L., \& Loren, C. (2010). La formación continua como proceso clave en la profesionalización docente: Buenas prácticas en Chile. Revista Latinoamericana de Educación Inclusiva, 4(1), 25-44. http://www. rinace.net/rlei/numeros/vol4-num1/art1.html

Moliner, O. (2008). Condiciones, procesos y circunstancias que permiten avanzar hacia la inclusion educativa: Retomando las aportaciones de la experiencia canadiense. Revista Electrónica Iberoamericana sobre Calidad,Eficaciay CambioenEducación,6(2),27-44.https://repositorio.uam.es/handle/10486/661085

Organización de las Naciones Unidas (1989). Convención de los Derechos del niño. https://www.ohchr.org/sp/ professionalinterest/pages/crc.aspx

Organización de las Naciones Unidas (2015). Agenda 2030 para el Desarrollo Sostenible. https://www.un.org/ sustainabledevelopment/es/development-agenda/

Organización de las Naciones Unidas para la Educación, la Ciencia y la Cultura. (2009). Directrices sobre políticas de inclusión en la educación. https://unesdoc.unesco.org/ark:/48223/pf0000177849_spa

Organización de las Naciones Unidas para la Educación, la Ciencia y la Cultura. (1994). Conferencia mundial sobre necesidades educativas especiales: Acceso y calidad - informe final. http://sid.usal.es/libros/ discapacidad/1366/8-4-2/conferencia-mundial-sobre-necesidades-educativas-especiales-informefinal-salamanca-espana-7-10-de-junio-1994.aspx

Powell, J. (2015). Barriers to inclusion: Special education in the United States and Germany. Routledge.

Sotomayor, C., \& Dupriez, V. (2007). Desarrollar competencias docentes en la escuela: Aprendizajes de una experiencia chilena de asesoría a escuelas de alta vulnerabilidad social y educativa. Les Cabiers de Recherche en Éducation et Formation, 61, 389-409. https://halshs.archives-ouvertes.fr/ halshs-00561937/document

Zhou, Y. (2012). Out-of-field teaching: A cross-national study on teacher labor market and teacher quality. [Tesis de Doctorado]. Facultad de Educación, Universidad Estatal de Michigan. https://eric. ed.gov/?id=ED548401

\section{Nota sobre autoria}

Conceptualización de la investigación - Benavides-Moreno N., Ortiz-González G. y Reyes-Araya D.; marco teórico - Benavides-Moreno N., Ortiz-González G. y Reyes-Araya D.; conclusiones - Benavides-Moreno N., Ortiz-González G. y Reyes-Araya D.

\section{Disponibilidad de datos}

Los datos de esta investigación están disponibles en: https://data.scielo.org/dataverse/brcp

\section{Cómo citar este artículo}

Benavides-Moreno, N., Ortiz-Gonzalez, G., \& Reyes-Araya, D. (2021). La inclusión escolar en Chile: Observada desde la docencia. Cadernos de Pesquisa, 51, Artículo e06806. https://doi. org/10.1590/198053146806

Recibido el: 4 SEPTIEMBRE 2019 | Aprobado para publicación el: 18 NOVIEMBRE 2020 